\title{
ARE TRANSFORMATIONAL AND ETHICAL LEADERSHIP TWO SIDES OF THE SAME COIN?
}

\author{
JUNGHYUN LEE \\ University of Michigan-Dearborn \\ College of Business \\ Fairlane Center South 19000 Hubbard Drive \\ Dearborn, Michigan 48126 \\ RAJNANDINI "RAJ" PILLAI \\ California State University San Marcos
}

\begin{abstract}
Using a longitudinal design in a non-western context, this study found that transformational leadership at Time 1 positively influenced followers' perceptions of ethical leadership at Time 2, mediated by procedural justice. Further, this mediated relationship was moderated by followers' power-distance orientation.
\end{abstract}

\section{INTRODUCTION}

Various corporate scandals over the last two decades have increased the importance and urgency of researching and developing ethical leadership. Accordingly, there has been growing interest in ethical leadership in the management literature. Prior research has shown that ethical leadership has a positive impact on followers' work-related attitudes, task performance, and organizational citizenship behavior (Brown, Trevino, \& Harrison, 2005; Piccolo, Greenbaum, Den Hartog, \& Folger, 2010; Walumbwa, Mayer, Wang, Wang, Workman, \& Christensen, 2011). Given the positive effect of ethical leadership, research on antecedents of becoming ethical leaders, such as individuals' personalities, motivation, and moral identity (Brown \& Trevion, 2006; Mayer, Aquino, Greenbaum, \& Kuenzi, 2012), provides useful insight to organizations by highlighting the importance of identifying potential leaders who possess these personal traits in a selection process. In order to further develop ethical leadership within the organization after leaders are selected, understanding what influences followers to view their leaders as ethical leaders is critical. With a goal of recognizing more proximate factors affecting ethical leadership, this study centers on the effects of transformational leadership on ethical leadership through the mediating role of procedural justice and also examines the moderating role of power distance in the relationship between ethical leadership and employee outcomes.

\section{THEORY AND HYPOTHESES}

\section{Transformational Leadership and Procedural Justice}

Transformational leaders' behaviors, especially those that relate to individualized consideration and inspirational motivation communicate to employees that they are valued by the authority figure of the organization (Tyler \& Lind, 1992). According to the relational model (Tyler, 1989; Tyler \& Lind, 1992), people are concerned about their long-term relationship with their organization and leaders, instead of viewing the relationship as a one-time deal. Further, 
people value civil treatment by groups and other parties they interact with because such treatment indicates that "they are valued members of the group who deserve treatment with respect, dignity, and politeness" (Tyler, 1989, p. 837). This, in turn, promotes their feelings of self-identity, self-esteem, and self-worth. As such, transformational leadership behavior is indicative of justice (Kuhnert \& Lewis, 1987), which in turn leads to positive attitudes toward work and organization. In addition, Transformational leaders may not only present ideal visions of the organization but also promote ethical policies, procedures, and processes (Howell \& Avolio, 1992). Communicating the policies of the organization makes a contribution to forming a culture of justice in employees (Niehoff \& Moorman, 1996). Thus, Pillai, Schriesheim, \& Williams (1999) argued that transformational leaders enhance individuals' perceptions of procedural justice by emphasizing the collective mission or vision and group solidarity. Similarly, Kim and colleagues (Kim, Kim, \& Kwon, 2010), in a study conducted in South Korea, found a positive relationship between transformational leadership and employees' procedural justice perceptions. Thus, we expect that when managers display behaviors consistent with transformational leadership, this will be positively associated with procedural justice (Kirkman, Chen, Farh, Chen, \& Lowe, 2009; Pillai et al., 1999).

\section{H1: Transformational leadership is positively related to procedural justice perceptions.}

Follower perceptions of procedural justice are closely related to ethical leadership as this aspect of justice is "based on prevailing ethical standards" (Cropanzano \& Ambrose, 2001, p.123). Literature on the psychology of procedural justice (Lind, 1995; Tyler, 1989) suggests that factors affecting judgments of procedural justice include having an unbiased decision maker who is honest and free from bias, using factual information to make decisions (neutrality), is concerned with others' interests (benevolence), and shows respect for individuals' rights as a group member (status recognition). Thus, fair treatment of followers is an important source of legitimacy for ethical leaders and legitimacy contributes to leaders' ability to gain voluntary compliance and support for decisions they make (Tyler, 1986). This suggests that procedural justice is an important element in judging leader's adherence to ethical leadership principles (Pillai, Scandura, \& Williams, 1999). Accordingly,

\section{H2: Procedural justice perceptions are positively related to ethical leadership.}

Followers come to trust leaders who treat them fairly, which in turn promotes followers' perceptions of ethical leadership because fair procedures enhance the impact of transformational leadership and lead to perceptions of ethical leadership (Pillai, Scandura, \& Williams, 1999). Followers realize that such leaders mean business in expecting ethical conduct from themselves and their subordinates. Consequently, followers believe that procedural justice is important in judging the adherence to ethical leadership principles by their leader (Pillai et. al, 1999). The relationship between transformational leadership and ethical leadership will be mediated by procedural justice as procedural justice is a central principle of ethical leaders manifested by fair and equal treatment of others (Johnson, 2009).

H3: Procedural justice perceptions mediate the relationship between transformational leadership and ethical leadership. 
Followers with a high power-distance orientation may accept their leader's actions uncritically without regard to perceptions of procedural justice (Lee, Pillutla, \& Law 2000). Thus, we argue that the positive relationship between procedural justice and ethical leadership will be weaker for followers with a high power-distance orientation. In contrast, followers with a low power-distance orientation tend to be egalitarian and perceive managers to be socially close. These followers would expect frequent and open communication with managers (Kirkman et al., 2009), which often facilitates the social learning process in the presence of ethical leadership (Brown et al., 2005; Salancik \& Pfeffer, 1978). As a result, low power-distance orientation may reinforce the link between procedural justice and ethical leadership, while high power-distance orientation may attenuate it. Therefore, we posit that the mediating effect of procedural justice will depend on followers' power-distance orientation (Farh, Hackett, \& Liang, 2007).

H4: Employees' power-distance orientation moderates the strength of the mediated relationship between transformational leadership and ethical leadership via procedural justice, such that the positive relationship is stronger when power-distance orientation is low rather than high.

Followers who form positive perceptions of how they are treated by ethical leaders (who exhibit honesty, trustworthiness, concern for employees, and their fair decision-making) may feel a sense of obligation to return the treatment in the same kind. Despite the paucity of research on ethical leadership conducted in a non-Western context, based upon the principle of social exchange perspective that was found to be generalized across nations and cultures, we expect the positive relationships between ethical leadership and follower outcomes to be replicated in the South Korean sample in the study.

H5: Ethical leadership is positively related to followers' job satisfaction, affective commitment, turnover intentions, and task performance.

Past research suggests that the positive influence of ethical leadership on follower attitudes and performance will be attenuated for followers with a high power-distance orientation because they tend to accept the imbalance in power and infrequently engage in two-way communication. This will result in followers with a high power distance orientation being less significantly affected by ethical leadership displayed by their supervisor (Lin, Wang, \& Chen 2013).

In contrast, when supervisors display low levels of ethical leadership, we predict that low power-distance followers will experience significantly decreased job satisfaction and affective commitment because they do not view leaders with the same level of reverence as high powerdistance followers. Similarly, these followers will more dramatically react to perceived low levels of ethical leadership by considering turnover and reducing task performance. Thus,

H6: Employees' power-distance orientation moderates the relationship between ethical leadership and employee outcomes, such that the positive relationship is stronger when powerdistance orientation is low rather than high.

\section{METHODS}

\section{Participants and Procedure}


We surveyed full-time, white-collar employees working at the headquarters of a manufacturing company in South Korea. Data were collected in three waves over a two-year period. Among 258 employees who were initially invited to the study, the final sample of 109 was used for analysis. The average age was 34 years $(\mathrm{SD}=5.8)$ and $71.9 \%$ of the participants were male. Supervisory tenure was 3.1 years on average $(\mathrm{SD}=3.4)$ and organizational tenure was 7.2 years $(S D=4.7)$ at Time 2 . Approximately $87 \%$ of participants had a college degree.

\section{Measures}

We translated psychometrically sound and widely used measures of transformational and ethical leadership, procedural justice, power-distance orientation, job satisfaction, affective commitment, and turnover intentions from English to Korean following Brislin's (1980) recommendations and followed up with a back translation. Employee performance was obtained from the Human Resources department of the company at Time 3.

\section{RESULTS}

Hierarchical linear regression analyses and boot strap analyses revealed that Hypotheses 1-4 were supported. Interaction plots show that the positive relationship between procedural justice and ethical leadership was stronger for individuals with a low power-distance orientation. Furthermore, the positive relationship between procedural justice and ethical leadership was stronger when power-distance orientation was low rather than high. With regard to Hypothesis 5 , ethical leadership was positively related to job satisfaction and affective commitment, marginally related to turnover intentions, but not related to task performance. For Hypothesis 6, power distance did moderate the relationship between ethical leadership and the three employee outcomes but not task performance.

\section{DISCUSSION}

Contributions of the current study are three-fold. First, our findings suggest that transformational leadership is an important antecedent to follower perceptions of their leaders as being ethical. While the leadership literature abounds with studies on the effects of various leadership styles on follower outcomes, there has been limited knowledge regarding how different leadership styles may relate to each other. The present study addresses this gap in the literature by incorporating the transformational leadership, ethical leadership, and procedural justice literatures. Second, the study demonstrates that procedural justice serves as an important linking pin between transformational leadership and ethical leadership. In particular, our approach of measuring ethical leadership one year after transformational leadership was measured enhances the level of confidence we can draw from the study. To the best of our knowledge, our study is the first one to examine the relationship between transformational leadership and ethical leadership using a longitudinal design. Third, our study contributes to the literature by examining the boundary condition by which promote or impede the effectiveness of leadership (Kacmar, Bachrach, Harris, \& Zivnuska, 2011; Kirkman et al., 2009; Walumbwa et al., 2011). In particular, understanding follower power-distance orientation can help leaders effectively predict followers' perceptions of and reactions to leadership behavior and accordingly adjust their behavior to further promote desired employee attitudes and performance. 
Ethical leadership is an important concern in contemporary organizations. So is changeoriented transformational leadership. Understanding how transformational leadership drives employee perceptions of fairness and ethical leadership allows organizations to harness the best aspects of both these leadership styles. In addition, leaders should pay special attention to their followers' receptivity to status and power. Our study suggests that managers need to pay attention to followers' power distance orientation that may enhance or attenuate the impact of leadership behavior on follower outcomes. As shown in the study, power-distance orientation affects the relationships between procedural justice and ethical leadership and between ethical leadership and employee outcomes. These findings shed light on the processes by which transformational leadership influences ethical leadership and the boundary conditions under which these relationships operate.

\section{REFERENCES AVAILABLE FROM THE AUTHORS}

